\title{
Can Corticospinal Excitability Shed Light Into the Effects of Handedness on Motor Performance?
}

\author{
Marco Antonio Cavalcanti Garcia ${ }^{1,2,3,4 *}$, Anaelli Aparecida Nogueira-Campos ${ }^{2,3}$, \\ Victor Hugo Moraes ${ }^{4,5}$ and Victor Hugo Souza ${ }^{3,6}$ \\ 1 Programa de Pós-Graduação em Ciências da Reabilitação e Desempenho Físico-Funcional, Faculdade de Fisioterapia, \\ Universidade Federal de Juiz de Fora, Juiz de Fora, Brazil, ${ }^{2}$ Laboratório de Neurofisiologia Cognitiva (LabNeuro), \\ Departamento de Fisiologia, Instituto de Ciências Biológicas, Universidade Federal de Juiz de Fora, Juiz de Fora, Brazil, \\ ${ }^{3}$ Grupo de Estudos em Neuro Biomecânica, Faculdade de Fisioterapia, Universidade Federal de Juiz de Fora, Juiz de Fora, \\ Brazil, ${ }^{4}$ Núcleo de Pesquisas em Neurociências e Reabilitação Motora, Universidade Federal do Rio de Janeiro, Rio de \\ Janeiro, Brazil, ${ }^{5}$ Instituto de Biofísica Carlos Chagas Filho, Universidade Federal do Rio de Janeiro, Rio de Janeiro, Brazil, \\ ${ }^{6}$ Department of Neuroscience and Biomedical Engineering, Aalto University School of Science, Espoo, Finland
}

Keywords: motor evoked potential, transcranial magnetic stimulation, lateral preference, handedness, motor skill

\section{OPEN ACCESS}

Edited by:

Wei-Peng Teo,

Nanyang Technological

University, Singapore

Reviewed by:

Florent Lebon,

INSERM U1093 Cognition, Action et Plasticité Sensomotrice, France

*Correspondence Marco Antonio Cavalcanti Garcia marco.garcia@ufj.edu.br; marco.garcia@ufff.br

Specialty section: This article was submitted to Physical Neuroergonomics,

a section of the journa Frontiers in Neuroergonomics

Received: 10 January 2021 Accepted: 01 March 2021 Published: 19 March 2021

Citation:

Garcia MAC, Nogueira-Campos AA, Moraes VH and Souza VH (2021) Can Corticospinal Excitability Shed Light Into the Effects of Handedness on Motor Performance?

Front. Neuroergon. 2:651501. doi: 10.3389/fnrgo.2021.651501

\section{INTRODUCTION}

Handedness is characterized as a lateral preference or advantage for one side of the body over the other when performing sensory or motor tasks (Cochet and Byrne, 2013). Different hypotheses have been formulated to explain this enigmatic human feature (Papadatou-Pastou et al., 2020). Genetic background (Annett, 1978; Paracchini and Scerri, 2017) anatomical or physiological asymmetries throughout the nervous system (NS) development or in adult life may contribute to the framework of lateral preference in primates (Daligadu et al., 2013). Understanding the origin of such asymmetries is crucial for the development of efficient patient-specific rehabilitation and training programs. Previous studies using neuroimaging (Amunts et al., 2000; Hervé et al., 2005) and neurophysiological techniques (Hammond et al., 2004; Souza et al., 2018) sought to quantify peripheral and central NS anatomical asymmetries using cadavers, for instance (White et al., 1994).

Alternatively, Transcranial Magnetic Stimulation (TMS) is a powerful tool to probe the brain function non-invasively in vivo. It has been used to unveil how the central NS drives the lateralization of motor gestures under different body postures on active and resting conditions (Triggs et al., 1994; Brouwer et al., 2001). TMS elicits motor evoked potentials (MEP) in a target muscle when delivered over the primary motor cortex (M1). Thus, monitoring changes in the MEP parameters provides a measure of corticospinal excitability (CSE) and relevant information about the integrity of the corticospinal tract (CST), especially in clinical conditions (Rossini et al., 2015). In the following topics, we describe recent findings and suitable recommendations for a more in-depth analysis of how CSE has been used for evaluating neurophysiologic attributes of handedness.

\section{ANATOMICAL FEATURES UNDERLYING THE CSE AND HANDEDNESS}

According to White et al. (1994), "humans have more cortical (and presumably subcortical) circuitry devoted to the representation of the right upper extremity than to the left." Thus, one might 
hypothesize that the CST would be larger on the right side of the spinal cord for right-handers. Likewise, the contrary stands true for left-handers even though the low prevalence limits the cadaveric approaches (9.3-18.1\%, Peters et al., 2006; Papadatou-Pastou et al., 2020). However, the reported results are inconclusive due to the limited number of studies conducted on cadavers. For instance, Yakovlev and Rakic (1966) and Melsbach et al. (1996) reported larger tracts in the right side of spinal cord of neonates and adult specimens. In contrast, Kertesz and Geschwind (1971) and White et al. (1997) did not observe any difference between both sides of medullae in 158 and 67 adult specimens, respectively. If the dominant side exhibits a larger CST, one might hypothesize that single-pulse TMS would recruit larger neuronal populations and, therefore, lead to higher MEP amplitudes in the dominant than the contralateral side. Indeed, few studies observed different MEP responses between dominant and non-dominant cerebral hemispheres (Triggs et al., 1994; Matsunaga et al., 1998).

Interestingly, asymmetries in the CST in neuroimaging studies (Ciccarelli et al., 2003; Westerhausen et al., 2007) or MEP amplitudes (Garcia et al., 2020) between both sides of the human body have been refuted. Alternatively, neuroimaging studies support the hypothesis that asymmetries in the right- and lefthanders take place at the cellular microstructure level throughout different NS regions, including the CST (Andersen and Siebner, 2018). Then humans are provided with more complex cortical and subcortical circuits devoted to benefit one side in specific motor tasks (Jang et al., 2017). Li et al. (2014) also suggest that handedness manifests in local neural networks due to "high local clustering and short paths between nodes" that can contribute to more significant asymmetries for right- but not left-handers. Their findings are partially supported by Hervé et al. (2005). They observed a positive correlation between contralateral gray matter volume and hand skills. It could be due to more complex neural networks with greater functional capacity. We might speculate that asymmetries in the CSE between the dominant and non-dominant sides observed in precision tasks (Triggs et al., 1994) were due to the extent of neural networks recruitment.

Consequently, CSE might better assess the degree of handedness when subjects are performing fine motor tasks, suggesting that some singularities of "dominant" neural networks recruited may be uncovered. Moreover, we should note that the TMS pulse recruits polysynaptic circuits mediated by inhibitory and excitatory interneurons and pyramidal neurons projecting into the CST (Di Lazzaro et al., 2018). Thus, one can argue that possible differences in MEP parameters from the dominant and non-dominant sides reside in the complexity of these high-order circuits engaged in performing motor tasks that do not require efforts above $\sim 10 \%$ of the maximal strength (Triggs et al., 1994) when compared the motor thresholds of different muscles from both upper limbs in the right- and left-handers. It was conducted during writing and under rest conditions. It is also suggested that motor tasks that require contractions above the mentioned level may normalize the cortical excitability and consequently mask differences in CSE (van de Ruit and Grey, 2016).

\section{METHODOLOGICAL APPROACHES ON MEP RECORDING APPLIED TO HANDEDNESS}

Three distinct MEP parameters are widely used to evaluate the CSE: latency, resting motor threshold (rMT) and peak-to-peak amplitude. Latency defines the onset time of the myoelectric activity evoked by the TMS pulse and may indicate pathological conditions such as demyelination (Fernández et al., 2013). Latency depends on the conduction velocity of the neural drive in the CST (Kidgell and Pearce, 2011). In this case, possible anatomical asymmetries associated with the ratio between fast and slow fibers would lead to distinct latencies from the dominant and non-dominant sides. Then, one may conjecture that the size principle could explain the differences in motor nerve latencies between both sides (Henneman et al., 1965). It is based on the concept that motor units containing axons with smaller diameters would have a lower conduction velocity and longer latencies. Curiously, most TMS studies did not report significant differences in MEP latencies in rest conditions between dominant and non-dominant sides (Kallioniemi et al., 2015), suggesting that latency in such condition may not be affected by manual dominance. Latency is frequently calculated by visual inspection and manually annotated in the surface electromyography (sEMG) recording. The manual annotation exhibits a high intrarater reliability (Livingston and Ingersoll, 2008) and the visual definition of onset time is subjective (Brown et al., 2017). Therefore, one should carefully interpret the physiological outcomes based on manually annotated latencies. An automated method was recently proposed by Šoda et al. (2020) and might be a better candidate to detect differences between dominant and non-dominant sides.

Likewise, asymmetries in the rMT have been used to understand how handedness manifests itself. The rMT represents the minimal TMS intensity delivered to M1 to elicit MEPs higher than a defined amplitude, e.g., $50 \mu \mathrm{V}$ (Rossini et al., 2015). Macdonell et al. (1991) reported lower rMT for the dominant cerebral hemispheres of right-handers. In contrast, Davidson and Tremblay (2013) recorded higher rMT in the dominant hemisphere of left-handed individuals. Remarkably, many other studies did not observe any significant rMT difference between both cerebral hemispheres (Livingston et al., 2010; Cueva et al., 2016; Garcia et al., 2020), refuting the previous findings. Even though Brouwer et al. (2001) also reported similar rMT between hands in right and left-handers, MEPs were higher when stronger hand muscles were recruited. It was hypothetically associated with the dominant side. Their findings also reinforce that MEP parameters related to the degree of handedness might be accessed when performing a motor task, as previously hypothesized.

Interestingly, a few authors reported other variables such as gender and age as possible intervening factors in handedness (Sala et al., 2017). In this respect, Amunts et al. (2000) reported anatomical asymmetries between cerebral hemispheres for righthanded males, but not females. In turn, Livingston et al. (2010) did not observe differences in MEP parameters when comparing both genders and right- and left-handers. According 
to Matsunaga et al. (1998), MEP parameters seem to be even less pronounced in older than young people.

MEP amplitude is associated with the level of motor unit recruitment. It may be affected by several factors such as postural adjustments (Sasaki et al., 2018), the type of task (rest vs. active) (Semmler and Nordstrom, 1998; Brouwer et al., 2001), and muscle length (Chye et al., 2010). Thus, methodological issues such as surface electrodes placement and the type of task performed, e.g., rest $v s$. active, may explain conflicting findings on CSE asymmetries between dominant and non-dominant sides (Triggs et al., 1994). For instance, Daligadu et al. (2013) observed an asymmetrical pattern on the stimulus-response curve between the dominant and nondominant cerebral hemispheres when TMS intensity was 90$150 \%$ of rMT. Interestingly, they found a lower rMT for the non-dominant side in right and left-handers, mainly for TMS intensities above $120 \%$ of rMT. This observation contrasts to the conventional idea that the dominant hand presents a lower level of excitability. The underlying mechanisms related to handedness under resting or active conditions may pose different views from the CSE and its anatomical and neurophysiological substrates. Souza et al. (2018) observed differences in MEP spatial distributions when using high-density sEMG, which were significantly shifted toward the lateral border of the thenar region on the dominant hand and might offer some advantage in generating torque in the metacarpal phalangeal joint. They suggest a biomechanical background on handedness according to the MEP spatial distribution and not solely on the amplitude. Therefore, alternative methodological approaches might provide additional insights into the effect of manual dominance on the CSE measures.

\section{THE MEASUREMENT OF THE DEGREE OF HANDEDNESS}

Questionnaires have been widely used to evaluate the degree of handedness (Oldfield, 1971). For instance, the Edinburgh Handedness Inventory (Oldfield, 1971) estimates the manual preference by a laterality quotient (LQ). Davidson and Tremblay (2013) observed that the LQ correlates with the degree of manual dexterity. Manual dexterity may also be associated with MEP parameters (Souza et al., 2018), which is postulated as derived from the degree of handedness. Nonetheless, there have been various adaptations on the original questionnaire (Edlin et al., 2015), which might have negatively impacted the agreement between different studies. Thus, the lack of uniformity or consensus in the use of Oldfield's and other questionnaires to determine the degree of laterality entangles the standardization of measurements and, consequently, the comparison between multiple studies. Flindall and Gonzalez (2019) go further on these issues, suggesting that many participants may report differences in the preferred hand for a task when asked on separate days for only a few weeks. Therefore, active motor tasks to quantify the degree of handedness seem to be a viable alternative (Cavill and Bryden, 2003; Flindall and Gonzalez, 2019). Indeed, a task-oriented evaluation may contribute to obtaining more clear discrimination of the degree of handedness, besides being correlated with anatomical and neurophysiology mechanisms. Registering the MEP while performing the motor tasks addressed by these questionnaires could contribute to evaluating the hypothetical relationship between the observed scores and the corticomotor pathway excitability.

\section{CONCLUSION}

The CSE might reveal underlying mechanisms that contribute to assess the degree of handedness, especially in active tasks. Moreover, MEPs might decode the central NS effort to drive a motor task, including biomechanical features, and, therefore, on differentiating degrees of handedness. Alternative methodological approaches combined with TMS, such as highdensity sEMG, might also contribute to discriminate handedness. Finally, the CSE might be a relevant measure to evaluate handedness while performing motor tasks but should be interpreted carefully due to the amount of variables involved.

\section{AUTHOR CONTRIBUTIONS}

MG, AAN-C, VM, and VS: conception, design, analysis, and interpretation of the literature. All authors have participated in drafting and critical revision of the manuscript for important intellectual content, final approval of the version to be submitted.

\section{FUNDING}

This study was financed in part by the Coordenação de Aperfeiçoamento de Pessoal de Nível Superior-Brasil (CAPES)_Finance Code 001, Fundação de Amparo à Pesquisa do Estado de São Paulo (FAPESP), Fundação de Amparo à Pesquisa do Estado de Minas Gerais (FAPEMIG). VS received funding from Conselho Nacional de Desenvolvimento Científico e Tecnológico (CNPq process number: 140787/2014-3), the Erasmus Mundus SMART2 (Project Reference: 552042-EM1-2014-1-FR-ERA MUNDUSEMA2), the Jane and Aatos Erkko Foundation, and from the European Research Council (ERC) under the European Union's Horizon 2020 research and innovation programme (grant agreement no. 810377). This research was also supported by Conselho Nacional de Desenvolvimento Científico e Tecnológico (CNPq process number: 426861/2016-7) assigned to AAN-C.

\section{ACKNOWLEDGMENTS}

The authors thank to Professor Ruben Ernesto de Bittencourt Navarrete (ICB/UFJF) for his careful English revision and substantial comments. 


\section{REFERENCES}

Amunts, K., Jäncke, L., Mohlberg, H., Steinmetz, H., and Zilles, K. (2000). Interhemispheric asymmetry of the human motor cortex related to handedness and gender. Neuropsychologia 38, 304-312. doi: 10.1016/S0028-3932(99)00075-5

Andersen, K. W., and Siebner, H. R. (2018). Mapping dexterity and handedness: recent insights and future challenges. Curr. Opin. Behav. Sci. 20, 123-129. doi: 10.1016/j.cobeha.2017.12.020

Annett, M. (1978). Genetic and nongenetic influences on handedness. Behav. Genet. 8, 227-249. doi: 10.1007/BF01072826

Brouwer, B., Sale, M., and Nordstrom, M. (2001). Asymmetry of motor cortex excitability during a simple motor task: relationships with handedness and manual performance. Exp. Brain Res. 138, 467-476. doi: 10.1007/s002210100730

Brown, K. E., Lohse, K. R., Mayer, I. M. S., Strigaro, G., Desikan, M., Casula, E. P., et al. (2017). The reliability of commonly used electrophysiology measures. Brain Stimul. 10, 1102-1111. doi: 10.1016/j.brs.2017.07.011

Cavill, S., and Bryden, P. (2003). Development of handedness: comparison of questionnaire and performance-based measures of preference. Brain Cogn. 53, 149-151. doi: 10.1016/S0278-2626(03)00098-8

Chye, L., Nosaka, K., Murray, L., Edwards, D., and Thickbroom, G. (2010). Corticomotor excitability of wrist flexor and extensor muscles during active and passive movement. Hum. Mov. Sci. 29, 494-501. doi: 10.1016/j.humov.2010.03.003

Ciccarelli, O., Toosy, A. T., Parker, G. J. M., Wheeler-Kingshott, C. A. M., Barker, G. J., Miller, D. H., et al. (2003). Diffusion tractography based group mapping of major white-matter pathways in the human brain. Neuroimage 19, 1545-1555. doi: 10.1016/S1053-8119(03)00190-3

Cochet, H., and Byrne, R. W. (2013). Evolutionary origins of human handedness: evaluating contrasting hypotheses. Anim. Cogn. 16, 531-542. doi: 10.1007/s10071-013-0626-y

Cueva, A. S., Galhardoni, R., Cury, R.G., et al. (2016). Normative data of cortical excitability measurements obtained by transcranial magnetic stimulation in healthy subjects. Neurophysiol. Clin. 46, 43-51. doi: 10.1016/j.neucli.2015.12.003

Daligadu, J., Murphy, B., Brown, J., Rae, B., and Yielder, P. (2013). TMS stimulusresponse asymmetry in left- and right-handed individuals. Exp. Brain Res. 224, 411-416. doi: 10.1007/s00221-012-3320-4

Davidson, T., and Tremblay, F. (2013). Hemispheric differences in corticospinal excitability and in transcallosal inhibition in relation to degree of handedness. PLoS ONE 8:e70286. doi: 10.1371/journal.pone.0070286

Di Lazzaro, V., Rothwell, J., and Capogna, M. (2018). Noninvasive stimulation of the human brain: activation of multiple cortical circuits. Neuroscientist 24, 246-260. doi: 10.1177/1073858417717660

Edlin, J. M., Leppanen, M. L., Fain, R. J., Hackländer, R. P., Hanaver-Torrez, S. D., and Lyle, K. B. (2015). On the use (and misuse?) of the edinburgh handedness inventory. Brain Cogn. 94, 44-51. doi: 10.1016/j.bandc.2015.01.003

Fernández, V., Valls-Sole, J., Relova, J. L., Raguer, N., Miralles, F., Dinca, L., et al. (2013). Recommendations for the clinical use of motor evoked potentials in multiple sclerosis. Neurología 28, 408-416. doi: 10.1016/j.nrleng.2013.09.003

Flindall, J. W., and Gonzalez, C. L. (2019). Wait wait, don't tell me: Handedness questionnaires do not predict hand preference for grasping. Laterality 24, 176-196. doi: 10.1080/1357650X.2018.1494184

Garcia, M. A. C., Souza, V. H., Lindolfo-Almas, J., Matsuda, R. H., and NogueiraCampos, A. A. (2020). Motor potential evoked by transcranial magnetic stimulation depends on the placement protocol of recording electrodes: a pilot study. Biomed. Phys. Eng. Express 6:047003. doi: 10.1088/2057-1976/ab950a

Hammond, G., Faulkner, D., Byrnes, M., Mastaglia, F., and Thickbroom, G. (2004). Transcranial magnetic stimulation reveals asymmetrical efficacy of intracortical circuits in primary motor cortex. Exp. Brain Res. 155, 19-23. doi: 10.1007/s00221-003-1696-x

Henneman, E., Somjen, G., and Carpenter, D. O. (1965). Excitability and inhibitibility of motoneurons of different sizes. J. Neurophysiol. 28, 599-620. doi: 10.1152/jn.1965.28.3.599

Hervé, P. Y., Mazoyer, B., Crivello, F., Perchey, G., and Tzourio-Mazoyer, N. (2005). Finger tapping, handedness and grey matter amount in the Rolando's genu area. Neuroimage 25, 1133-1145. doi: 10.1016/j.neuroimage.2004.12.062
Jang, H., Lee, J. Y., Lee, K. I., and Park, K. M. (2017). Are there differences in brain morphology according to handedness? Brain Behav. 7:e00730. doi: 10.1002/brb3.730

Kallioniemi, E., Pitkänen, M., Säisänen, L., and Julkunen, P. (2015). Onset latency of motor evoked potentials in motor cortical mapping with neuronavigated transcranial magnetic stimulation. Open Neurol. J. 9, 62-69. doi: 10.2174/1874205X01509010062

Kertesz, A., and Geschwind, N. (1971). Patterns of Pyramidal Decussation and Their Relationship to Handedness. Arch. Neurol. 24, 326-332. doi: 10.1001/archneur.1971.00480340058006

Kidgell, D. J., and Pearce, A. J. (2011). What has transcranial magnetic stimulation taught us about neural adaptations to strength training? A brief review. J. Strength Cond. Res. 25, 3208-3217. doi: 10.1519/JSC.0b013e318212de69

Li, M., Chen, H., Wang, J., Liu, F., Long, Z., Wang, Y., et al. (2014). Handednessand hemisphere-related differences in small-world brain networks: a diffusion tensor imaging tractography study. Brain Connect. 4, 145-156. doi: 10.1089/brain.2013.0211

Livingston, S. C., Goodkin, H. P., and Ingersoll, C. D. (2010). The influence of gender, hand dominance, and upper extremity length on motor evoked potentials. J. Clin. Monit. Comput. 24, 427-436. doi: 10.1007/s10877-010-9267-8

Livingston, S. C., and Ingersoll, C. D. (2008). Intra-rater reliability of a transcranial magnetic stimulation technique to obtain motor evoked potentials. Int. J. Neurosci. 118, 239-256. doi: 10.1080/00207450701668020

Macdonell, R. A. L., Shapiro, B. E., Chiappa, K. H., Helmers, S. L., Cros, D., Day, B. J., et al. (1991). Hemispheric threshold differences for motor evoked potentials produced by magnetic coil stimulation. Neurology 41, 1441-1441. doi: 10.1212/WNL.41.9.1441

Matsunaga, K., Uozumi, T., Tsuji, S., and Murai, Y. (1998). Age-dependent changes in physiological threshold asymmetries for the motor evoked potential and silent period following transcranial magnetic stimulation. Electroencephalogr. Clin. Neurophysiol. 109, 502-507. doi: 10.1016/S1388-2457(98)00020-0

Melsbach, G., Wohlschläger, A., Spieß, M., and Güntürkün, O. (1996). Morphological Asymmetries of motoneurons innervating upper extremities: clues to the anatomical foundations of handedness? Int. J. Neurosci. 86, 217-224. doi: 10.3109/00207459608986712

Oldfield, R. C. (1971). The assessment and analysis of handedness: the edinburgh inventory. Neuropsychologia 9, 97-113. doi: 10.1016/0028-3932(71)90067-4

Papadatou-Pastou, M., Ntolka, E., Schmitz, J., Martin, M., Munafò, M. R., Ocklenburg, S., et al. (2020). Human handedness: a meta-analysis. Psychol. Bull. 146, 481-524. doi: 10.1037/bul0000229

Paracchini, S., and Scerri, T. (2017). "Genetics of Human Handedness and Laterality", in Lateralized Brain Functions. Neuromethods, eds L. Rogers and G. Vallortigara (New York, NY: Humana Press), 523-552. doi: 10.1007/978-1-4939-6725-4_16

Peters, M., Reimers, S., and Manning, J. T. (2006). Hand preference for writing and associations with selected demographic and behavioral variables in 255,100 subjects: the BBC internet study. Brain Cogn. 62, 177-189. doi: 10.1016/j.bandc.2006.04.005

Rossini, P. M., Burke, D., Chen, R., Cohen, L. G, Daskalakis, Z., Di Iorio, R., et al. (2015). Non-invasive electrical and magnetic stimulation of the brain, spinal cord, roots and peripheral nerves: basic principles and procedures for routine clinical and research application. An updated report from an I.F.C.N. Committee. Clin. Neurophysiol. 126, 1071-1107. doi: 10.1016/j.clinph.2015.02.001

Sala, G., Signorelli, M., Barsuola, G., Bolognese, M., and Gobet, F. (2017). The relationship between handedness and mathematics is non-linear and is moderated by gender, age, and type of task. Front. Psychol. 8:948. doi: 10.3389/fpsyg.2017.00948

Sasaki, A., Milosevic, M., Sekiguchi, H., and Nakazawa, K. (2018). Evidence for existence of trunk-limb neural interaction in the corticospinal pathway. Neurosci. Lett. 668, 31-36. doi: 10.1016/j.neulet.2018.01.011

Semmler, J. G., and Nordstrom, M. A. (1998). Hemispheric differences in motor cortex excitability during a simple index finger abduction task in humans. $J$. Neurophysiol. 79, 1246-1254. doi: 10.1152/jn.1998.79.3.1246

Šoda, J., Vidaković, M. R., Lorincz, J., Jerković, A., and Vujović, I. (2020). A novel latency estimation algorithm of motor evoked potential signals. IEEE Access. 8, 193356-193374. doi: 10.1109/ACCESS.2020.3033075 
Souza, V. H., Baffa, O., and Garcia, M. A. C. (2018). Lateralized asymmetries in distribution of muscular evoked responses: an evidence of specialized motor control over an intrinsic hand muscle. Brain Res. 1684, 60-66. doi: 10.1016/j.brainres.2018.01.031

Triggs, W. J., Calvanio, R., Macdonell, R. A. L., Cros, D., and Chiappa, K. H. (1994). Physiological motor asymmetry in human handedness: evidence from transcranial magnetic stimulation. Brain Res. 636, 270-276. doi: 10.1016/0006-8993(94)91026-X

van de Ruit, M., and Grey, M. J. (2016). The TMS map scales with increased stimulation intensity and muscle activation. Brain Topogr. 29, 56-66. doi: 10.1007/s10548-015-0447-1

Westerhausen, R., Huster, R. J., Kreuder, F., Wittling, W., and Schweiger, E. (2007). Corticospinal tract asymmetries at the level of the internal capsule: is there an association with handedness? Neuroimage 37, 379-386. doi: 10.1016/j.neuroimage.2007.05.047

White, L. E., Andrews, T. J., Hulette, C., Richards, A., Groelle, M., Paydarfar, J., et al. (1997). Structure of the human sensorimotor system. II: Lateral symmetry. Cereb. Cortex 7, 31-47. doi: 10.1093/cercor/7.1.31
White, L. E., Lucas, G., Richards, A., and Purves, D. (1994). Cerebral asymmetry and handedness. Nature 368, 197-198. doi: 10.1038/36 $8197 \mathrm{a} 0$

Yakovlev, P. I., and Rakic, P. (1966). Patterns of decussation of bulbar pyramids and distribution of pyramidal tracts on two sides of the spinal cord. Trans. Am. Neurol. Assoc. 91, 366-367.

Conflict of Interest: The authors declare that the research was conducted in the absence of any commercial or financial relationships that could be construed as a potential conflict of interest.

Copyright (C) 2021 Garcia, Nogueira-Campos, Moraes and Souza. This is an openaccess article distributed under the terms of the Creative Commons Attribution License (CC BY). The use, distribution or reproduction in other forums is permitted, provided the original author(s) and the copyright owner(s) are credited and that the original publication in this journal is cited, in accordance with accepted academic practice. No use, distribution or reproduction is permitted which does not comply with these terms. 